\title{
Nuclear composition and heating in accreting neutron-star crusts
}

\author{
P. Haensel and J. L. Zdunik
}

\author{
N. Copernicus Astronomical Center, Polish Academy of Sciences, Bartycka 18, 00-716 Warszawa, Poland \\ e-mail: jlz@camk.edu.pl
}

Received 11 March 2003 / Accepted 12 May 2003

\begin{abstract}
Nuclear reactions in accreting neutron-star crusts and the heat release accompanying them are studied, under different assumptions concerning the composition of the outermost layer formed of the ashes of X-ray bursts. Particular examples of ashes containing nuclides with $A \simeq 90-110$ are considered and compared with a standard $A=56$ case. In all cases, evolution of a crust shell is followed from $10^{8} \mathrm{~g} \mathrm{~cm}^{-3}$ to a few times $10^{13} \mathrm{~g} \mathrm{~cm}^{-3}$. The total crustal heating produced in the non-equilibrium processes in the accreting crust is $1.1-1.5 \mathrm{MeV}$ per one accreted nucleon. The composition of the accreted crust at densities exceeding the threshold for the pycnonuclear fusion $\left(\rho>10^{12} \mathrm{~g} \mathrm{~cm}^{-3}\right)$ is essentially independent of the assumed initial composition of the X-ray burst ashes.
\end{abstract}

Key words. dense matter - equation of state - stars: neutron - stars :general - X-rays: bursts - X-ray: binaries nuclear reactions

\section{Introduction}

Accretion of matter onto neutron stars in close binary systems plays crucial role in many objects studied by the X-ray astronomy, such as X-ray bursters, X-ray pulsars, and transient X-ray sources. Particular attention was focused recently on soft Xray transients (SXRTs) in quiescence, when the accretion from a disk formed of matter flowing from the low-mass companion star is switched off or strongly suppressed. The nature of the X-ray radiation during quiescence is still a matter of debate. Typically, the quiescent emission is much higher than the expected one for an old cooling neutron star. It has been suggested that this is due to the fact that the interiors of neutron stars in SXRTs are heated-up, during relatively short periods of accretion and bursting, by the non-equilibrium processes associated with nuclear reactions taking place in the deep layers of the crust (Brown et al. 1998). This additional crustal heating, combined with appropriate models of neutron-star atmosphere and interior, can be used to explain observations of SXRTs in quiescence. Dependence of the luminosity in quiescence on the structure of neutron-star core, and particularly on the rate of neutrino cooling, opened a new possibility of exploring the internal structure and equation of state of neutron stars via confrontation of theoretical models with observations of quiescent SQRTs (see, e.g., Ushomirsky \& Rutledge 2001; Colpi et al. 2001; Rutledge et al. 2002; Brown et al. 2002, and references therein).

The crustal heating used in the SQRTs models was described using the results of the calculations of Haensel \& Zdunik (1990) (hereafter referred to as HZ). In the HZ model,

Send offprint requests to: P. Haensel, e-mail: haensel@camk . edu.pl an outer layer of neutron star, formed in X-ray bursts, is composed of ${ }^{56} \mathrm{Fe}$. Such a composition, corresponding to the maximum nuclear binding, was predicted by the standard models of the thermonuclear X-ray bursts of that time. Then, the ${ }^{56} \mathrm{Fe}$ layer is sinking in the neutron star interior under the weight of accreted matter. Under increasing pressure, the composition of matter is changing in a sequence of nuclear reactions: electron captures, neutron emission and absorption, and finally, at densities exceeding $10^{12} \mathrm{~g} \mathrm{~cm}^{-3}$, also pycnonuclear fusion. As the nuclear processes are proceeding off-equilibrium, they are accompanied by the heat deposition in the crustal matter. For the HZ model, the total crustal heating amounts to $1.4 \mathrm{MeV}$ per one accreted nucleon. This heating is mostly supplied by the pycnonuclear fusion processes in the inner crust at $\rho=10^{12}-10^{13} \mathrm{~g} \mathrm{~cm}^{-3}$.

Very recently, new X-ray burst simulations, with a network of nuclei much larger than the previously used networks which ended with the iron-peak nuclei, were carried out by Schatz et al. (2001). Extending the network of possible nuclear reactions resulted in nuclear ashes composed predominantly of nuclei much heavier than iron, with $A$ up to $\sim 112$. The question arises how changing the initial composition from ${ }^{56} \mathrm{Fe}$ to, e.g., ${ }^{104} \mathrm{Te}$ will change the crustal heating and the composition of the deeper layers of the inner crust. The present Letter is devoted to answering this question.

Brief description of the dense-matter model and of the processes taking place in an accreting neutron-star crust is given in Sect. 2. Results for the composition and heating, obtained for various initial compositions and dense-matter models, are presented in Sect. 3. Final Sect. 4 is devoted to a discussion of our results and conclusion. 


\section{Non-equilibrium nuclear processes}

In what follows we briefly describe the nuclear evolution scenario of $\mathrm{HZ}$ as applied in the present study (earlier studies on various aspects of the non-equilibrium neutron-star crusts were done by Vartanyan \& Ovakimova 1976, Bisnovatyi-Kogan \& Chechetkin 1978, Sato 1979). Under conditions prevailing in accreting neutron-star crust at $\rho>10^{8} \mathrm{~g} \mathrm{~cm}^{-3}$ matter is strongly degenerate, and is relatively cold $\left(T<10^{8} \mathrm{~K}\right)$, so that thermonuclear processes involving charged particles are blocked. At the densities lower than the threshold for the pycnonuclear fusion $\rho_{\text {pyc }} \sim 10^{12} \mathrm{~g} \mathrm{~cm}^{-3}$, the number of nuclei in an element of matter does not change during the compression resulting from the increasing weight of accreted matter. For simplicity, we assume that only one nuclear species $(A, Z)$ is present at each pressure. Due to the nucleon pairing, stable nuclei in dense matter have even $N=A-Z$ and $Z$ (even-even nuclides). In the outer crust, in which free neutrons are absent, the electron captures which proceed in two steps,

$(A, Z)+\mathrm{e}^{-} \longrightarrow(A, Z-1)+v_{\mathrm{e}}$

$(A, Z-1)+\mathrm{e}^{-} \longrightarrow(A, Z-2)+v_{\mathrm{e}}+Q_{\mathrm{c}}$

lead to a systematic decrease of $Z$ (and increase of $N=A-Z$ ) with increasing density. The first capture in Eq. (1) proceeds in a quasi-equilibrium manner, with a negligible energy release. It produces an odd-odd nucleus, which is strongly unstable in dense medium, and captures a second electron in an nonequilibrium manner, with energy release $Q_{\mathrm{c}}$. A fraction of this energy, $Q_{\mathrm{d}}$, is deposited in the matter and heats it, but most of it, $Q_{\nu}$, is carried away by the neutrino, so that $Q_{\mathrm{c}}=Q_{\mathrm{d}}+Q_{\nu}$. The effective heat deposited in matter is

$Q_{\mathrm{d}}=\eta\left(\mu_{\mathrm{e}}-\Delta\right)+Q_{\mathrm{exc}}$,

where $\mu_{\mathrm{e}}$ is the electron Fermi energy (including the rest energy) and $\Delta$ is the energy threshold for the first quasiequilibrium electron capture. $Q_{\mathrm{exc}}$ is the excitation energy of the final nucleus, and $\eta$ ranging from $1 / 6$ to $1 / 4$ accounts for the neutrino energy losses. We put $Q_{\text {exc }}=0$ in the actual simulations, except for one case with initial ${ }^{56} \mathrm{Fe}$ composition when $Q_{\text {exc }}$ is experimentally known (see HZ). We have $\eta=1 / 6$ for $\mu_{\mathrm{e}} \gg \Delta$ and $\eta=1 / 4$ for $\left(\mu_{\mathrm{e}}-\Delta\right) / \mu_{\mathrm{e}} \ll 1$. In the crust evolution simulation the latter condition is fulfilled and therefore we put $\eta=1 / 4$. Above the neutron-drip point $\left(\rho>\rho_{\mathrm{ND}}\right)$, electron captures trigger neutron emissions,

$(A, Z)+\mathrm{e}^{-} \longrightarrow(A, Z-1)+v_{\mathrm{e}}$

$(A, Z-1)+\mathrm{e}^{-} \longrightarrow(A-k, Z-2)+k \mathrm{n}+v_{\mathrm{e}}+Q_{\mathrm{c}}$

where the number of emitted neutrons " $k$ " is even. Due to the electron captures, the value of $Z$ decreases with increasing density. In consequence, the Coulomb barrier prohibiting the nucleus-nucleus reaction lowers. This effect, combined with the decrease of the separation between the neighboring nuclei, and a simultaneous increase of energy of the quantum zeropoint vibrations around the nuclear lattice sites, opens a possibility of the pycnonuclear reactions. The pycnonuclear fusion timescale $\tau_{\text {pyc }}$ is a very sensitive function of $Z$. The chain of the reactions (3) leads to an abrupt decrease of $\tau_{\text {pyc }}$ typically by 7 to 10 orders of magnitude. Pycnonuclear fusion switcheson as soon as $\tau_{\text {pyc }}$ is smaller than the time of the travel of a piece of matter (due to the accretion) through the considered shell of mass $M_{\text {shell }}(N, Z), \tau_{\text {acc }} \equiv M_{\text {shell }} / \dot{M}$. The masses of the shells are of the order of $10^{-5} M_{\odot}$. As a result the point where the pycnonuclear reaction takes place is very well defined and the chain of reactions (3) in several cases is followed by the pycnonuclear reaction on a timescale much shorter than $\tau_{\text {acc }}$. Denoting $Z^{\prime}=Z-2$, we have then

$$
\begin{aligned}
& \left(A, Z^{\prime}\right)+\left(A, Z^{\prime}\right) \longrightarrow\left(2 A, 2 Z^{\prime}\right)+Q_{1}, \\
& \left(2 A, 2 Z^{\prime}\right) \longrightarrow\left(2 A-k^{\prime}, 2 Z^{\prime}\right)+k^{\prime} \mathrm{n}+Q_{2}, \\
& \ldots \quad \ldots \quad \ldots \quad \ldots+Q_{3},
\end{aligned}
$$

where "..." correspond to some not specified chain of the electron captures accompanied by neutron emissions. The total heat deposition in matter, resulting from a chain of reactions involving a pycnonuclear fusion, is $Q_{\mathrm{pyc}}=Q_{1}+Q_{2}+Q_{3, \mathrm{~d}}$ where label "d" indicates that only the fraction deposited in the matter is included.

Our model of atomic nuclei is described in HZ. Using our nuclear-evolution code, we evolved an element of matter consisting initially of nuclei $\left(A_{\mathrm{i}}, Z_{\mathrm{i}}\right)$ immersed in an electron gas, from $\rho_{\mathrm{i}}=10^{8} \mathrm{~g} \mathrm{~cm}^{-3}$ to $\rho=\rho_{\mathrm{f}}>5 \times 10^{13} \mathrm{~g} \mathrm{~cm}^{-3}$. Our results for the composition and crustal heating are presented in the next section.

\section{Composition and heating}

The compositions of accreted neutron star crusts are shown in Fig. 1 and in Table 1. These results describe crusts built of accreted and processed matter up to the density $5 \times 10^{13} \mathrm{~g} \mathrm{~cm}^{-3}$. At a constant accretion rate $\dot{M}=\dot{M}_{-9} \times 10^{-9} M_{\odot} /$ yr this will take $\sim 10^{6} \mathrm{yr} / \dot{M}_{-9}$. During this time, a shell of X-ray burst ashes will be compressed from $\sim 10^{8} \mathrm{~g} \mathrm{~cm}^{-3}$ to $\sim 10^{13} \mathrm{~g} \mathrm{~cm}^{-3}$. Two different compositions of X-ray bursts ashes at $\lesssim 10^{8} \mathrm{~g} \mathrm{~cm}^{-3}$ were assumed. In the first case, $A_{\mathrm{i}}=56$, and the $\mathrm{HZ}$ scenario is reproduced. In the second case, we consider an example of nuclear ashes obtained by Schatz et al. (2001). To be specific, we assume $A_{\mathrm{i}}=106$. The value of $Z_{\mathrm{i}}=46$ stems then from the condition of beta equilibrium at $\rho=10^{8} \mathrm{~g} \mathrm{~cm}^{-3}$. The compositions in the outer crust, where the only processes are the electron captures, are strongly influenced by the initial conditions. Up to the neutron-drip point, the difference by a factor of about two between the values of $Z$ and $N$ for $A_{\mathrm{i}}=106$ and $A_{\mathrm{i}}=56$ is conserved. It should be noted that in the case $A_{\mathrm{i}}=106$ the number of beta captures in the outer crust is about 2 times larger, but each reaction is accompanied by the density jump $\sim 5 \%$, about half of these in the case $A_{\mathrm{i}}=56$ (we have similar situation for the energy release in a single shell). One notices a dramatic effect of the neutron drip triggered by an electron capture at $\rho=\rho_{\mathrm{ND}}$. We get $\rho_{\mathrm{ND}}=6 \times 10^{11} \mathrm{~g} \mathrm{~cm}^{-3}$ for $A_{\mathrm{i}}=56$ and $\rho_{\mathrm{ND}}=8 \times 10^{11} \mathrm{~g} \mathrm{~cm}^{-3}$ for $A_{\mathrm{i}}=106$. Just after $\rho_{\mathrm{ND}}$ both $N$ and $Z$ of nuclei decrease in a long chain of the neutron emissions followed by the electron captures. After the pycnonuclear fusion is switched-on at $\rho_{\text {pyc }} \simeq 10^{12} \mathrm{~g} \mathrm{~cm}^{-3}$, 

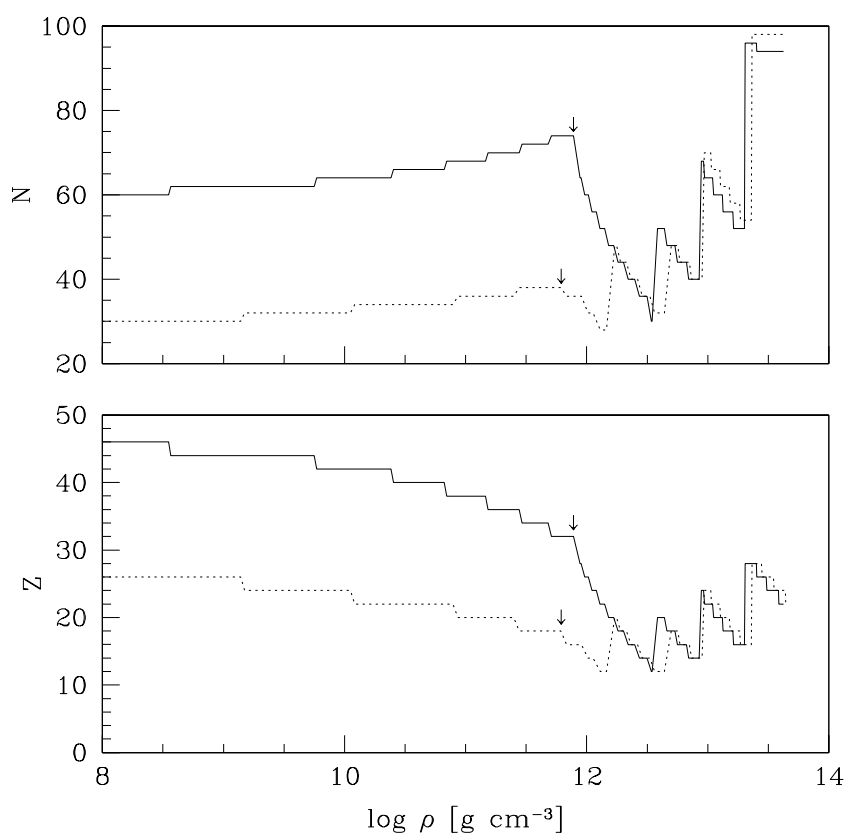

Fig. 1. $Z$ and $N$ of nuclei versus matter density in an accreting neutronstar crust. Solid line: $A_{\mathrm{i}}=106$; dotted line: $A_{\mathrm{i}}=56$. Each change of $N$ and $Z$, which takes place at a constant pressure, is accompanied by a jump in density (see HZ for detailed discussion of this point). Small steep segments connect the top and the bottom density of thin reaction shell. Arrows indicate positions of the neutron drip point.

the two compositions converge, and stay very close, up to the largest densities beyond which the validity of the HZ model becomes questionable. We checked that this is a generic property of the $Z, N$ evolution, which does not depend on specific values of $A_{\mathrm{i}}, Z_{\mathrm{i}}$, or on the details of the nuclear model used (see Sect. 4).

In Fig. 2 we show the heat deposited in the matter, per one accreted nucleon, in the thin shells in which non-equilibrium nuclear processes are taking place. Actually, reactions proceed at a constant pressure, and there is a density jump within a thin "reaction shell". The vertical lines whose height gives the heat deposited in matter are drawn at the density at the bottom of the reaction shell.

One notices a specific dependence of the number of heat sources and the heating power of a single source on assumed $A_{\mathrm{i}}$. Let us start with the outer crust (upper panel of Fig. 2). In the case of $A_{\mathrm{i}}=56$ the number of sources is smaller, and their heat-per-nucleon values $q$ are larger, than for $A_{\mathrm{i}}=106$. Hovever, the total deposited heat-per-nucleon is quite similar, 0.041 and $0.039 \mathrm{MeV} /$ nucleon for $A_{\mathrm{i}}=56$ and $A_{\mathrm{i}}=106$, respectively. Similar features are seen in the inner crust (lower panel of Fig. 2). The total crustal heating is $1.54 \mathrm{MeV} /$ nucleon and $1.12 \mathrm{MeV} /$ nucleon for $A_{\mathrm{i}}=56$ and $A_{\mathrm{i}}=106$, respectively. The difference $\simeq 0.4 \mathrm{MeV} /$ nucleon between these two cases is mainly due to an additional pycnonuclear reaction (the first one) in the case $A_{\mathrm{i}}=56$, which results in the convergence of two evolutionary scenarios at $\rho \simeq 2 \times 10^{12} \mathrm{~g} \mathrm{~cm}^{-3}$. This pycnonuclear fusion is accompanied by the larger energy release than the subsequent beta captures and neutron emissions in the case $A_{\mathrm{i}}=106$. The nearly exact convergence of
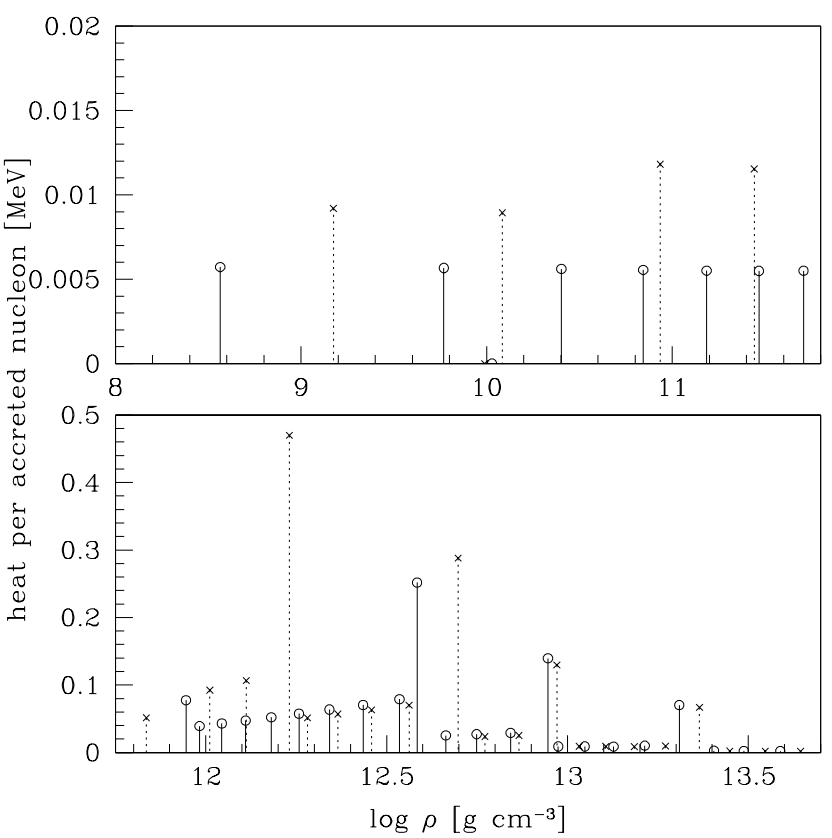

Fig. 2. Heat per one accreted nucleon, deposited in the crust, for two models with different initial $A$. Solid vertical lines (ended with circles): $A_{\mathrm{i}}=106$; dotted lines (ended with crosses): $A_{\mathrm{i}}=56$. Vertical lines are positioned at the densities at the bottom of the reaction shell.

the cases $A_{\mathrm{i}}=56$ and $A_{\mathrm{i}}=106$ for $\rho>10^{12} \mathrm{~g} \mathrm{~cm}^{-3}$ is connected with the fact that heavier nucleus has $N_{\mathrm{i}}$ and $Z_{\mathrm{i}}$ which are nearly double of those of ${ }^{56} \mathrm{Fe}$. In the case of the initial nuclei between $A_{\mathrm{i}}=56$ and $A_{\mathrm{i}}=106$ the situation is similar with the nearly same nuclei after the first pycnonuclear reaction, with the slight shift in the densities of the boundaries between subsequent shells. The total energy per nucleon released then above the neutron drip point is between 1.1 and $1.5 \mathrm{MeV}$, with unchanged crustal heating below the neutron drip. The value obtained for $A_{\mathrm{i}}=56$ is slightly larger than that quoted in $\mathrm{HZ}$, which results from correcting too large neutrino losses in electron captures assumed in HZ.

\section{Discussion and conclusion}

We studied nuclear composition of accreted neutron-star crusts, assuming different compositions of the X-ray bursts ashes. Obtained compositions $(A, Z)$ of the outer crust for ashes with $A_{\mathrm{i}}=56$ and $A_{\mathrm{i}} \simeq 100$ keep the difference by a factor of about two up to the neutron-drip point. However, total crustal heating in the outer crust is similar for both $A_{\mathrm{i}}$, and is negligible compared with the crustal heating in the inner crust. A chain of processes occurring after the neutron drip leads to convergence of compositions to a common one, which at densities higher than $10^{12} \mathrm{~g} \mathrm{~cm}^{-3}$ does not depend on $A_{\mathrm{i}}$. While the number of the heat-sources depends on $A_{\mathrm{i}}$, and is significantly larger for $A_{\mathrm{i}} \simeq 100$ than for $A_{\mathrm{i}}=56$, total crustal heating, $1.1 \mathrm{MeV} /$ nucleon and $1.5 \mathrm{MeV} /$ nucleon, is quite similar. Simulations with other values of $A_{\mathrm{i}}$ confirm that the crustal heating is only moderately dependent on the nuclear composition of the X-ray bursts ashes.

Let us mention, that in a very recent paper Schatz et al. (2003) suggest that rp-processes ashes can burn explosively at 
Table 1. Non-equilibrium processes in the crust of an accreting neutron stars assuming that the X-ray ashes consist of ${ }^{106} \mathrm{Pd} . P$ and $\rho$ are pressure and density at which the reaction takes place. $\Delta \rho / \rho$ is relative density jump connected with reaction, $q$ is the heat deposited in matter, $X_{\mathrm{n}}$ is the fraction of free neutrons among nucleons, in the layer just above the reaction surface.

\begin{tabular}{|c|c|c|c|c|c|}
\hline $\begin{array}{l}P \\
\left({\left.\text { dyn } \mathrm{cm}^{-2}\right)}\right)\end{array}$ & $\begin{array}{l}\rho \\
\left(\mathrm{g} \mathrm{cm}^{-3}\right)\end{array}$ & reactions & $X_{\mathrm{n}}$ & $\begin{array}{r}\Delta \rho / \rho \\
\%\end{array}$ & $\begin{array}{r}q \\
(\mathrm{keV})\end{array}$ \\
\hline $9.235 \times 10^{25}$ & $3.517 \times 10^{08}$ & ${ }^{106} \mathrm{Pd} \rightarrow{ }^{106} \mathrm{Ru}-2 \mathrm{e}^{-}+2 v_{\mathrm{e}}$ & 0 & 4.4 & 5.7 \\
\hline $3.603 \times 10^{27}$ & $5.621 \times 10^{09}$ & ${ }^{106} \mathrm{Ru} \rightarrow{ }^{106} \mathrm{Mo}-2 \mathrm{e}^{-}+2 v_{\mathrm{e}}$ & 0 & 4.6 & 5.7 \\
\hline $2.372 \times 10^{28}$ & $2.413 \times 10^{10}$ & ${ }^{106} \mathrm{Mo} \rightarrow{ }^{106} \mathrm{Zr}-2 \mathrm{e}^{-}+2 v_{\mathrm{e}}$ & 0 & 4.9 & 5.6 \\
\hline $8.581 \times 10^{28}$ & $6.639 \times 10^{10}$ & ${ }^{106} \mathrm{Zr} \rightarrow{ }^{106} \mathrm{Sr}-2 \mathrm{e}^{-}+2 v_{\mathrm{e}}$ & 0 & 5.1 & 5.6 \\
\hline $2.283 \times 10^{29}$ & $1.455 \times 10^{11}$ & ${ }^{106} \mathrm{Sr} \rightarrow{ }^{106} \mathrm{Kr}-2 \mathrm{e}^{-}+2 v_{\mathrm{e}}$ & 0 & 5.4 & 5.5 \\
\hline $5.025 \times 10^{29}$ & $2.774 \times 10^{11}$ & ${ }^{106} \mathrm{Kr} \rightarrow{ }^{106} \mathrm{Se}-2 \mathrm{e}^{-}+2 v_{\mathrm{e}}$ & 0 & 5.7 & 5.5 \\
\hline $9.713 \times 10^{29}$ & $4.811 \times 10^{11}$ & ${ }^{106} \mathrm{Se} \rightarrow{ }^{106} \mathrm{Ge}-2 \mathrm{e}^{-}+2 v_{\mathrm{e}}$ & 0 & 6.1 & 5.5 \\
\hline $1.703 \times 10^{30}$ & $7.785 \times 10^{11}$ & ${ }^{106} \mathrm{Ge} \rightarrow{ }^{92} \mathrm{Ni}+14 \mathrm{n}-4 \mathrm{e}^{-}+4 v_{\mathrm{e}}$ & 0.13 & 13.2 & 77.6 \\
\hline $1.748 \times 10^{30}$ & $8.989 \times 10^{11}$ & ${ }^{92} \mathrm{Ni} \rightarrow{ }^{86} \mathrm{Fe}+6 \mathrm{n} 2 \mathrm{e}^{-}+2 v_{\mathrm{e}}$ & 0.19 & 6.9 & 39.2 \\
\hline $1.924 \times 10^{30}$ & $1.032 \times 10^{12}$ & ${ }^{86} \mathrm{Fe} \rightarrow{ }^{80} \mathrm{Cr}+6 \mathrm{n} 2 \mathrm{e}^{-}+2 v_{\mathrm{e}}$ & 0.25 & 7.3 & 43.1 \\
\hline $2.135 \times 10^{30}$ & $1.197 \times 10^{12}$ & ${ }^{80} \mathrm{Cr} \rightarrow{ }^{74} \mathrm{Ti}+6 \mathrm{n} 2 \mathrm{e}^{-}+2 v_{\mathrm{e}}$ & 0.30 & 7.7 & 47.4 \\
\hline $2.394 \times 10^{30}$ & $1.403 \times 10^{12}$ & ${ }^{74} \mathrm{Ti} \rightarrow{ }^{68} \mathrm{Ca}+6 \mathrm{n} 2 \mathrm{e}^{-}+2 v_{\mathrm{e}}$ & 0.36 & 8.1 & 52.3 \\
\hline $2.720 \times 10^{30}$ & $1.668 \times 10^{12}$ & ${ }^{68} \mathrm{Ca} \rightarrow{ }^{62} \mathrm{Ar}+6 \mathrm{n} 2 \mathrm{e}^{-}+2 v_{\mathrm{e}}$ & 0.42 & 8.5 & 57.7 \\
\hline $3.145 \times 10^{30}$ & $2.016 \times 10^{12}$ & ${ }^{62} \mathrm{Ar} \rightarrow{ }^{56} \mathrm{~S}+6 \mathrm{n} 2 \mathrm{e}^{-}+2 v_{\mathrm{e}}$ & 0.47 & 9.0 & 63.7 \\
\hline $3.723 \times 10^{30}$ & $2.488 \times 10^{12}$ & ${ }^{56} \mathrm{~S} \rightarrow{ }^{50} \mathrm{Si}+6 \mathrm{n} 2 \mathrm{e}^{-}+2 v_{\mathrm{e}}$ & 0.53 & 9.4 & 70.5 \\
\hline $4.549 \times 10^{30}$ & $3.153 \times 10^{12}$ & ${ }^{50} \mathrm{Si} \rightarrow{ }^{42} \mathrm{Mg}+8 \mathrm{n} 2 \mathrm{e}^{-}+2 v_{\mathrm{e}}$ & 0.61 & 8.8 & 79.0 \\
\hline $4.624 \times 10^{30}$ & $3.472 \times 10^{12}$ & $\begin{array}{l}{ }^{42} \mathrm{Mg} \rightarrow{ }^{36} \mathrm{Ne}+6 \mathrm{n} 2 \mathrm{e}^{-}+2 v_{\mathrm{e}} \\
{ }^{36} \mathrm{Ne}+{ }^{36} \mathrm{Ne} \rightarrow{ }^{72} \mathrm{Ca}\end{array}$ & 0.66 & 10.6 & 251.8 \\
\hline $5.584 \times 10^{30}$ & $4.399 \times 10^{12}$ & ${ }^{72} \mathrm{Ca} \rightarrow{ }^{66} \mathrm{Ar}+6 \mathrm{n} 2 \mathrm{e}^{-}+2 v_{\mathrm{e}}$ & 0.69 & 4.8 & 25.3 \\
\hline $6.883 \times 10^{30}$ & $5.355 \times 10^{12}$ & ${ }^{66} \mathrm{Ar} \rightarrow{ }^{60} \mathrm{~S}+6 \mathrm{n} 2 \mathrm{e}^{-}+2 v_{\mathrm{e}}$ & 0.72 & 4.7 & 27.3 \\
\hline $8.749 \times 10^{30}$ & $6.655 \times 10^{12}$ & ${ }^{60} \mathrm{~S} \rightarrow{ }^{54} \mathrm{Si}+6 \mathrm{n} 2 \mathrm{e}^{-}+2 v_{\mathrm{e}}$ & 0.75 & 4.6 & 29.2 \\
\hline $1.157 \times 10^{31}$ & $8.487 \times 10^{12}$ & $\begin{array}{l}{ }^{54} \mathrm{Si} \rightarrow{ }^{46} \mathrm{Mg}+8 \mathrm{n} 2 \mathrm{e}^{-}+2 v_{\mathrm{e}} \\
{ }^{46} \mathrm{Mg}+{ }^{46} \mathrm{Mg} \rightarrow{ }^{92} \mathrm{Cr}\end{array}$ & 0.79 & 4.0 & 139.6 \\
\hline $1.234 \times 10^{31}$ & $9.242 \times 10^{12}$ & ${ }^{92} \mathrm{Cr} \rightarrow{ }^{86} \mathrm{Ti}+6 \mathrm{n} 2 \mathrm{e}^{-}+2 v_{\mathrm{e}}$ & 0.80 & 2.0 & 8.9 \\
\hline $1.528 \times 10^{31}$ & $1.096 \times 10^{13}$ & ${ }^{86} \mathrm{Ti} \rightarrow{ }^{80} \mathrm{Ca}+6 \mathrm{n}^{2} \mathrm{e}^{-}+2 v_{\mathrm{e}}$ & 0.82 & 1.9 & 9.0 \\
\hline $1.933 \times 10^{31}$ & $1.317 \times 10^{13}$ & ${ }^{80} \mathrm{Ca} \rightarrow{ }^{74} \mathrm{Ar}+6 \mathrm{n} 2 \mathrm{e}^{-}+2 v_{\mathrm{e}}$ & 0.83 & 1.8 & 8.8 \\
\hline $2.510 \times 10^{31}$ & $1.609 \times 10^{13}$ & ${ }^{74} \mathrm{Ar} \rightarrow{ }^{68} \mathrm{~S}+6 \mathrm{n} 2 \mathrm{e}^{-}+2 v_{\mathrm{e}}$ & 0.85 & 1.7 & 10.2 \\
\hline $3.363 \times 10^{31}$ & $2.003 \times 10^{13}$ & $\begin{array}{l}{ }^{68} \mathrm{~S} \rightarrow{ }^{62} \mathrm{Si}+6 \mathrm{n} 2 \mathrm{e}^{-}+2 v_{\mathrm{e}} \\
{ }^{62} \mathrm{Si}+{ }^{62} \mathrm{Si} \rightarrow{ }^{124} \mathrm{Ni}\end{array}$ & 0.86 & 1.7 & 70.3 \\
\hline $4.588 \times 10^{31}$ & $2.520 \times 10^{13}$ & ${ }^{124} \mathrm{Ni} \rightarrow{ }^{120} \mathrm{Fe}+4 \mathrm{n} 2 \mathrm{e}^{-}+2 v_{\mathrm{e}}$ & 0.87 & 0.8 & 2.6 \\
\hline $5.994 \times 10^{31}$ & $3.044 \times 10^{13}$ & ${ }^{120} \mathrm{Fe} \rightarrow{ }^{118} \mathrm{Cr}+2 \mathrm{n} 2 \mathrm{e}^{-}+2 v_{\mathrm{e}}$ & 0.88 & 0.9 & 2.4 \\
\hline $8.408 \times 10^{31}$ & $3.844 \times 10^{13}$ & ${ }^{118} \mathrm{Cr} \rightarrow{ }^{116} \mathrm{Ti}+2 \mathrm{n} 2 \mathrm{e}^{-}+2 v_{\mathrm{e}}$ & 0.88 & 0.8 & 2.2 \\
\hline
\end{tabular}

density $\sim 10^{9} \mathrm{~g} \mathrm{~cm}^{-3}$, giving rise to the X-ray superburst. Such a burning will lead to photodisintegration of the $A \sim 100$ nuclei and shift composition of the ashes toward iron, so that in this case the original scenario of $\mathrm{HZ}$ will hold.

In view of the importance of the crustal heating for the SXRTs models, more detailed study of non-equilibrium processes in accreting neutron-star crusts is desirable. An investigation along these lines is now being carried out and its results will be presented in a separate publication (Haensel \& Zdunik, in preparation).

Acknowledgements. We are very grateful to the referee for detailed and helpful comments. We are also grateful to D.G. Yakovlev for useful correspondence. This work was supported in part by the KBN grant 5 P03D 02020.

\section{References}

Bildsten, L., \& Chakrabarty, D. 2001, ApJ, 557, 292

Bisnovatyi-Kogan, G. S., \& Chechetkin, V. M. 1979, Uspekhi Fiz. Nauk, 127, 263 (English translation: Sov. Phys. Uspekhi, 22, 89)

Brown, E. F., Bildsten, L., \& Rutledge, R. E. 1998, ApJ, 504, L95

Brown, E. F., Bildsten, L., \& Chang, P. 2002, ApJ, 574, 920

Campana, S., Stella, L., Gastaldello, F., et al. 2002, ApJ, 575, L15

Colpi, M., Geppert, U., Page, D., \& Possenti, A. 2001, ApJ, 548, L175

Chen, W., Shrader, C. R., \& Livio, M. 1997, ApJ, 491, 312

Haensel, P., \& Zdunik, J. L. 1990, A\&A, 227, 431

Sato, K. 1979, Prog. Theor. Phys., 62, 957

Schatz, H., Aprahamian, A., Barnard, V., et al. 2001, Phys. Rev. Lett., 86,3471

Schatz, H., Bildsten, L., \& Cumming, A. 2003, ApJ, 583, L87

Vartanyan, Yu. L., \& Ovakimova, N. K. 1976, Soobtcheniya Byurakanskoi Observatorii, 49, 87 